\title{
Optimal integration of pneumatic artificial muscles with vacuum-jammed surfaces to characterise a novel reconfigurable moulding system
}

\author{
Hossein Habibi, Patrick Land, Michael J. Ball, David Alatorre Troncoso, David T. Branson III \\ Advanced Manufacturing Technology Research Group, Faculty of Engineering, University of Nottingham,
} UK

\begin{abstract}
Producing mould tooling systems is a considerable proportion of manufacturing cost and time, especially for low volume productions. Producing a reconfigurable mould to shape surfaces into complex geometries with multiple curvatures would obviate the need to design and fabricate individual moulds for different products. Current reconfigurable mould tooling systems are mainly variations on a 'bed of pins' design through differing patterns of actuated pins. These systems are heavy, mechanically complex and expensive to manufacture. Soft pneumatic actuators such as Mckibben muscles, also known as pneumatic artificial muscles (PAMs) are recognised for their high strength to weight ratio, ease of manufacture and low cost. In this work, PAMs are used to influence a soft elastomeric surface, allowing the formation of spatial curved profiles. This thin, hollow surface is packed with a granular medium that exhibits jamming under the negative pressure of a vacuum. This allows the flexible surface to transform to a rigid surface of greatly increased stiffness with a specific geometry for moulding purposes. This paper presents the design, experimental development and experimental performance of two sample prototypes to actualizes the idea of such adaptable moulding tools. The prototypes contain different actuator arrangements to form jamming surfaces into diverse, simple-to-complex 3D profiles. In addition, the kinematic performance for one of the prototypes is shown by a numerical model. Future work on this system will tailor it for production of fibre carbon composites.
\end{abstract}

Keywords: Reconfigurable mould; vacuum jammed surface; pneumatic artificial muscle; soft tooling.

\section{Introduction}

A reconfigurable mould that can be used to shape parts of differing geometries is a highly desirable prospect in a number of areas of manufacturing. The most important reason for this is the factor of cost as the construction of these parts, often of limited number of uses before a new mold is required, can represent a significant proportion of the total cost per part, especially for low volume productions. Additionally, related costs are also incurred for storage, maintenance and logistics of using a large number of different moulds. This is particularly true for the aerospace industry, with tooling representing over $10 \%$ of the cost per part in some cases [1]. The development of a low-cost solution that reduces the need for these conventional moulds has many potential benefits to both the aerospace industry and numerous other manufacturing areas. Previous work in the area of reconfigurable tooling has primarily consisted of reconfigurable pinmatrix based systems such as that presented in the works of Peters and Marion [2] and Koc et al [3]. Most commonly used in the incremental forming of sheet metal structures, these systems consist of a number of individual pins, actuated in a variety of manners which can be individually positioned to create a 3-dimensional replication of the input. Use of an interpolator layer is necessary to maintain an acceptable surface profile. Common methods of actuation utilised in 
these systems include: shaft driven lead screws, hydraulic actuation and sequential pin set up methods [4]. Problems with current reconfigurable tooling methods such as complexity, cost and difficulty in obtaining high tolerances due to the nature of the interpolator layer, have prevented the uptake of this technology and have limited the application of a reconfigurable tool within areas such as composites processing [5]. In the context of improving such systems, there are several further studies that mainly present the use of various mechanisms for actuated discrete pins with application to sheet metal forming [6-8]. However, the fundamental problems with these systems in terms of cost and complexity remain.

An area that provides the starting point for an alternative to the discrete pin based systems in the work discussed above is the newly emerging technology of granular jamming. Media such as sand, beads or grains contained within a volume, exhibit the reversible phenomenon of jamming when subjected to compressive stress such as the negative pressure of a vacuum. This allows vacuum-jammed volumes to transition between a flexible, reconfigurable state and a pseudo-solid, jammed state, in which it exhibits increased stiffness and an ability to hold its shape [9]. This characteristic of tunable stiffness gives rise to the concept of a jammable surface which can form shapes when flexible and then increase stiffness to lock in place. The relative stiffness of vacuum jammed surfaces is a product of a number of factors. At comparative levels of compressive stress, the stiffness is governed by the relative motion ability of the granular medium. This is dependent on particle shape, size, material and homogeneity and surface texture [10]. The potential for exploitation of this controllable phase change has been explored in a work by Steltz et al [11] on the use of jamming as an enabling technology for soft robotics. The concept presented involved utilising vacuum-jammed structural elements as a method of achieving locomotion in a mobile robot. This work illustrates the potential for use of the vacuum jamming principles within the context of a controllable volume and highlights manufacturing techniques which have proved successful in the creation of an effective vacuum-jammed surface.

Consideration of the actuation methods for a vacuum-jammed surface included the review of solutions previously utilised in reconfigurable systems such as those discussed above. These included shape memory alloys, electro-active polymers, tension cable systems and servo motors [4 and 12]. However, a more suitable alternative to these conventional, rigid actuation methods could be pneumatic artificial muscles (PAMs). These actuators, also commonly known as McKibben muscles were developed in artificial limb research in the 1960s and consist of an internal bladder surrounded by an expandable braided mesh sleeving fastened at either end [13]. Pressurizing the internal bladder causes an increase in volume of the muscle in a direction prescribed by the nature of the braided mesh. Conventional PAMs expand circumferentially causing an axial contraction [14]. However they can also be made to extend axially reducing in diameter. The soft nature of these actuators allows them to produce interesting non-linear motions when arranged or joined in specific ways, where for the purposes of a reconfigurable mould it is desirable to produce surface curvature. Combining two extensible PAMs in parallel in a fashion analogous to a bi-metallic strip or a pair of agonist-antagonist muscles allows the creation of a curving actuator set. Where the extended PAM forces deformation around a bend to maintain continuity at the fixed end points. This ability to produce uniform curvature, combined with the high strength to weight ratio, soft nature and low cost of these actuators make them an attractive choice for use within a reconfigurable surface over methods such as shape memory alloys, electroactive polymers, tension cable systems and servo motors.

This paper looks to an optimal integration of these two technologies, granular jamming surfaces and PAMs, to present a prototype reconfigurable manufacturing mould. The nature of this lightweight and rapidly reconfigurable surface enables a variety of potential applications to industry. A primary focus for this technology is to integrate into the composite materials market, this industry often has a requirement for rapid mould geometries with single, double or multiple 
curvatures. The reconfigurable surface technology presents the potential to enable rapid development of bespoke composite components to industries such are aerospace, marine and energy technology, while providing a relatively low-cost and repeatable tooling method.

The developed prototypes will also underpin a significant step towards advancing the understanding and ability to design, model and control actuated Large Displacement Continuum Surfaces (LDCS) that have been used previously in soft robotic systems [15-18]. Although to date the integration of both an actuated deformable surface and on-demand surface stiffening system has not been done. In Section 2 the conceptual model of the integrated system will be presented. Section 3 will then illustrates the optimisation of the actuators through experimental characterisation of the parameters pressure, curvature, length and force. Section 4 presents aspects of design, fabrication and optimisation of the vacuum-jammed surfaces used in this work. Finally the development and performance of two prototype examples of the reconfigurable mould consisting of optimally characterised PMAs (one with 4 and the other with 16 PAMs) and an optimised vacuum-jammed surface to shape deformable composite sheets will be described in Section 5 .

\section{Conceptual model of an actuated deformable surface system}

The optimal integration of these two technologies (vacuum jamming and PAMs) into a unique solution to the problem of achieving a reconfigurable, cost-effective moulding system was inspired by examples of projects in the respective technological areas. It was also informed by prior art on reconfigurable mould solutions and the gap in the market for a low-cost solution that would reduce the significant cost of single-component tooling.

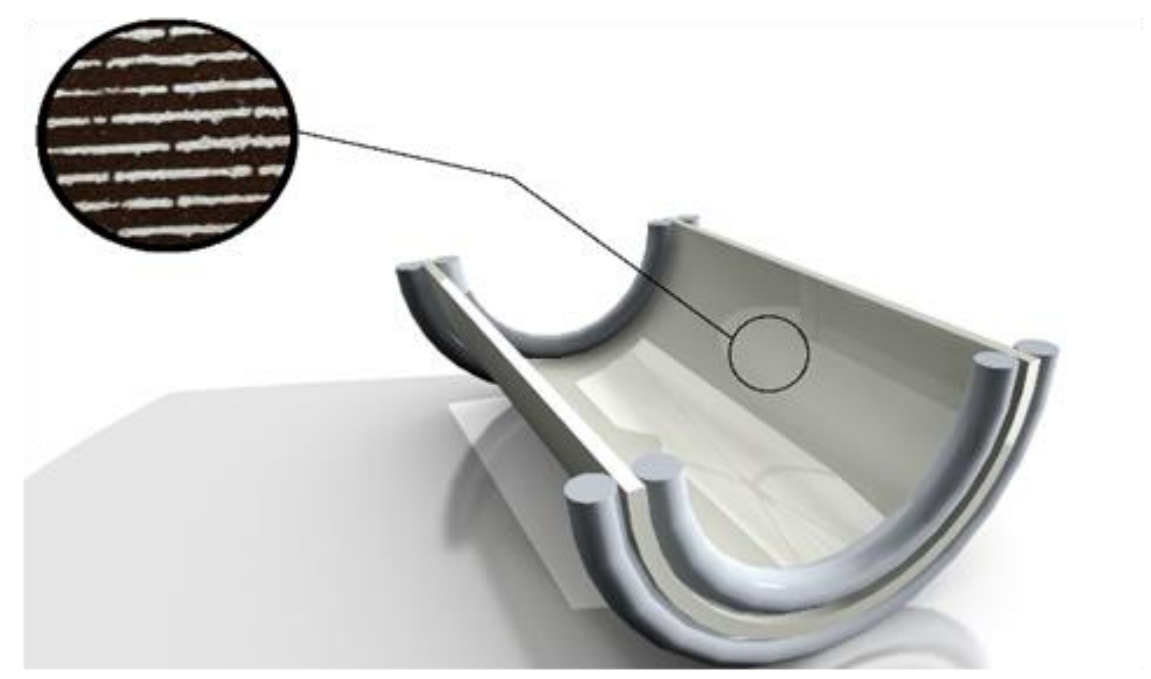

Fig. 1. A reconfigurable model concept with an internal view of the surface

The proposed concept involves a thin, flexible elastomeric surface with a densely packed granular media internal to this surface. The even distribution of this medium within the surface is maintained via division of the cavity into cells. This ensures that the granular medium will not move during deformation, before the vacuum is pulled. Sets of PAM actuators are integrated into the edges of the surface to allow formation of uniform curvature. Fig. 1 shows a model of the system concept with an enlarged internal view of the surface. In the model shown in Fig 1, only one axis of curvature is considered. However, multi-axis deformation can be achieved through the integration of actuators along additional axes or even across the surface. In the operation shown here the lower PAM of each actuator set is pressurised, causing it to extend and forcing the surface to deform around a curvature due to the lack of similar deformation by the upper PAM. 
When the desired shape is achieved, the air is removed from the surface, jamming the granular medium and forming a rigid shape that can then be used for moulding. As the performance of the system is dependent upon both the surface and the actuator capabilities, these two elements are studied and optimised separately before the integrated system's function will be presented in this work.

\section{Actuator optimisation}

Optimisation of the actuators involved modifications to the design of the PAMs to achieve operation up to 5 bars. Characterisation of the curvature and force abilities was achieved through experimentation with the optimised design.

\subsection{Design and fabrication of actuators}

As with typical PAMs, the design consists of an internal bladder and a braided mesh sleeving. Conventionally, PAMs will contract or expand when pressurised due to circumferential expansion of the internal bladder against the outer material creating force along the actuator's axis [19]. The actuation principles and detailed procedure for designing PAMs are well demonstrated in previous studies [13, 14, 20, and 21]. In this work, the actuators are manufactured with an $8 \mathrm{~mm}$ outer diameter platinum cured silicone bladder. This is covered with an expandable braided PET sleeving $(2-8 \mathrm{~mm})$ that is compressed to the length of the bladder from its original state.

The fabrication process for the PAMs can be also found well in literature [19, 20, and 21]. To measure the curvature of the actuator in this work, a $100-\mathrm{mm}$ flex sensor (produced by Spectrasymbol; Part ID: FS-L-0095-103-ST) was integrated into the actuator pair at the middle point. The performance of the flex sensor is based on resistive carbon elements (emerging as conductive ink) printed on a thin flexible substrate. When the substrate is bent, the sensor produces a resistance output correlated to the bend radius - the smaller the radius, the higher the resistance value - which can be easily measured.

Connecting two PAMs at the fittings and pressuring one causes the set to deform around a uniform curve as shown in Fig. 2. This occurs because the length of one PMA increases, while the other remains unchanged. Effectively forcing the extending actuator to 'curve' to maintain the restricted displacement of the non-extending actuator due to their connection at the ends, and further anchoring along their length. These lengthwise anchors also ensure continuous contact between the PAMs.

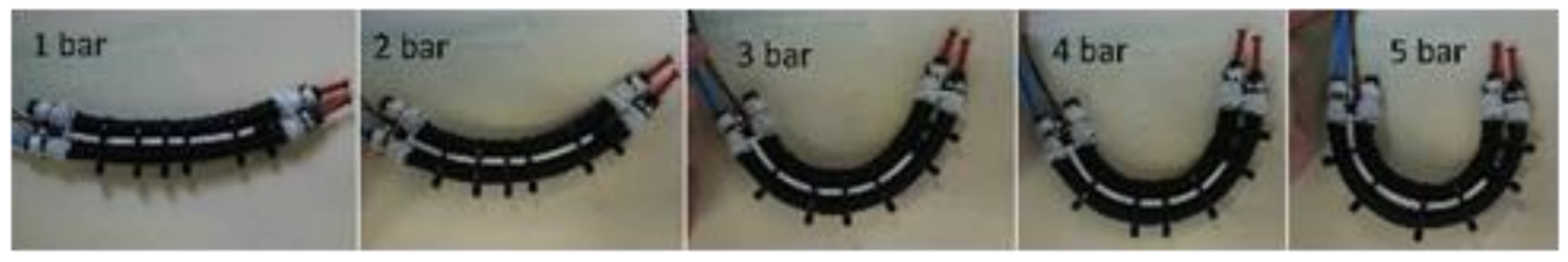

Fig. 2. Actuator deforming around uniform curve when bottom actuator is pressurized, causing it to extend.

\subsection{Experimental characterisation of actuators}

PAMs are hugely customisable actuators with a large number of varying designs [14]. The fact that they utilise a compressible gas results in much higher compliance than conventional rigid actuators. The behaviour of PAMs is therefore highly non-linear and difficult to characterize numerically. The studies by Chou and Hannaford [13] and Delson et al [19] among others has sought to model the performance of a number of different PAMs. However, to the author's knowledge no such theoretical work exists that directly corresponds with the modified design and 
configuration explored in this paper. As development of such a model was beyond the scope of this project, experimental work was undertaken to determine performance relationships for the design in question.

Experimentation sought to assess the relationships for pressure-curvature, length of actuatorcurvature and pressure-force output to determine the capabilities of the actuators and provide the information necessary to size actuators for specific requirements and applications. These experiments were carried out for 4 lengths of actuators, equally spaced in the range 50-200 mm. The results of the pressure-curvature experiments over a 5 bar pressure range, provided data for the length-curvature relationship and indicated the length required to achieve a desired curvature at a given operating pressure.

\subsubsection{Pressure - Curvature}

A 100-mm bending actuator was fabricated with a flex sensor of equal length secured between the PAMs, along the axis of curvature. Using a median averaged signal from this sensor, the experimental procedure involved increasing the gauge pressure of the actuator to 5 bar in increments of 0.2 bar and recording the resulting curvature of the actuator pair.

Due to limitations in available lengths of the flex sensors for shorter actuation elements, an alternate, visual method of determining curvature was developed. Figure 3 shows the experimental setup that was used to obtain visual information over the range of pressures for each actuator. Using the 3D CAD Software Creo, a circle was superimposed upon a photo of the curved actuator and the angle subtended between two reference points (the ends of the bladder) giving then the curvature of the actuator as shown in Fig. 4.

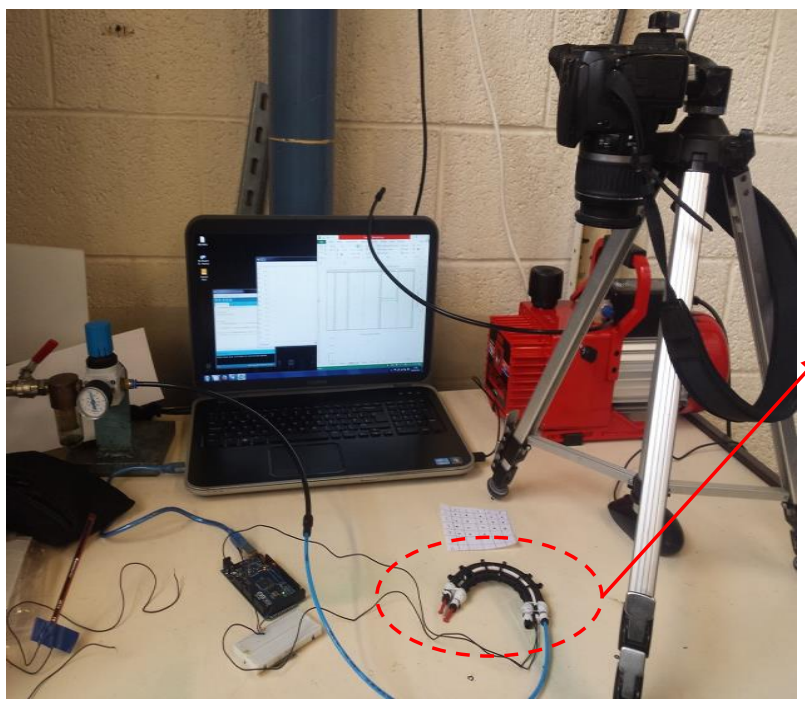

Fig. 3. Experimental setup: taking top-down photos of curvatures

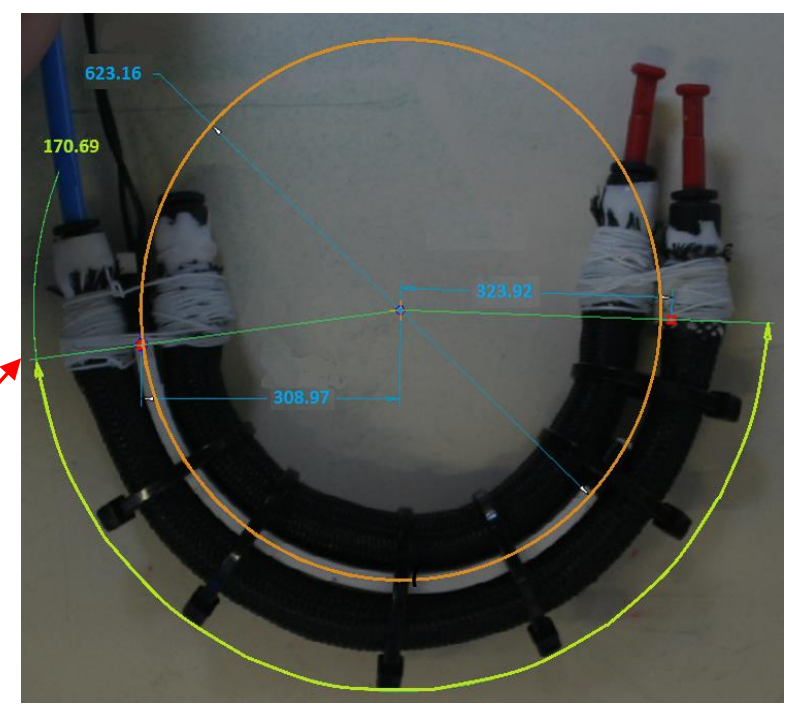

Fig.4. Graphical determination of curvature

To validate this method, the graphical data was compared against the flex sensor data as illustrated in Fig. 5 for the $100 \mathrm{~mm}$ actuator. The curvature values obtained using the graphical method (as used for all other length actuators) are shown alongside the values obtained via the integrated flex sensor. The clear agreement between these two data sets implies the reliable use of the graphical method as a means to determine curvature. 


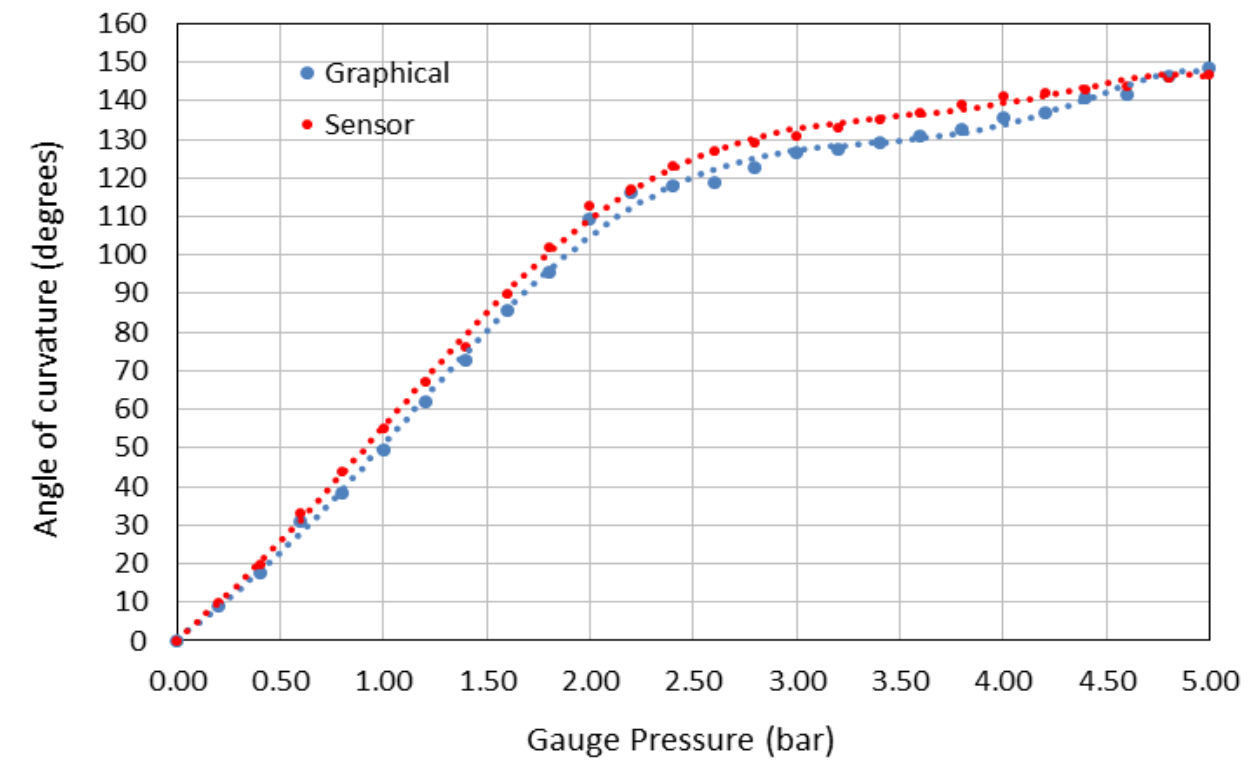

Fig. 5. Gauge pressure versus net angle of curvature for 100mm actuator; Comparison of flex sensor data and curvature values obtained via graphical method

Figure 6 shows the curvature (normalised by subtracting curvature at $\mathrm{P}=0$ ) for the four lengths of actuator across the operational pressure range (OPR). The non-linearity of the actuator response highlights the complexity of attempting to model PAMs theoretically. The increase in curvature can be seen to proceed at a reducing rate, plateauing as the actuators reach the upper end of the OPR. Below gauge pressures of 2 bar, an approximate linear relationship exists between the pressure and curvature for all 4 actuators. This data outlines the actuator capabilities and allows rough specification of the pressure required to form a desired curvature.

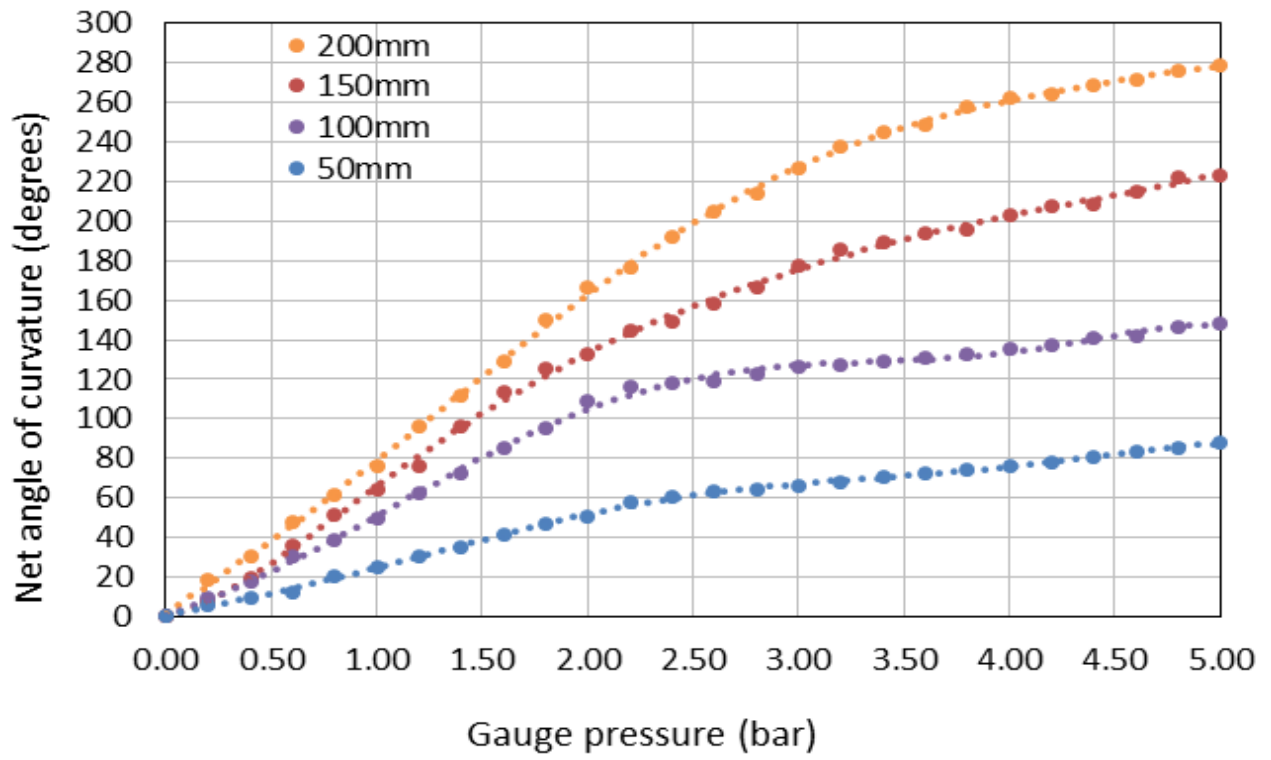

Fig. 6. Net angle of curvature formed by 4 actuator lengths across operational pressure range

\subsubsection{Length-Curvature}

At the top end of the OPR where the curvature increases have plateaued significantly, the relationship between curvature and length is almost linear as shown in Fig.7. These values are 
more useful for sizing the actuators to a given application as they give an underestimated indication of the maximum curvature achievable for a particular size of surface (into which the actuators will be integrated).

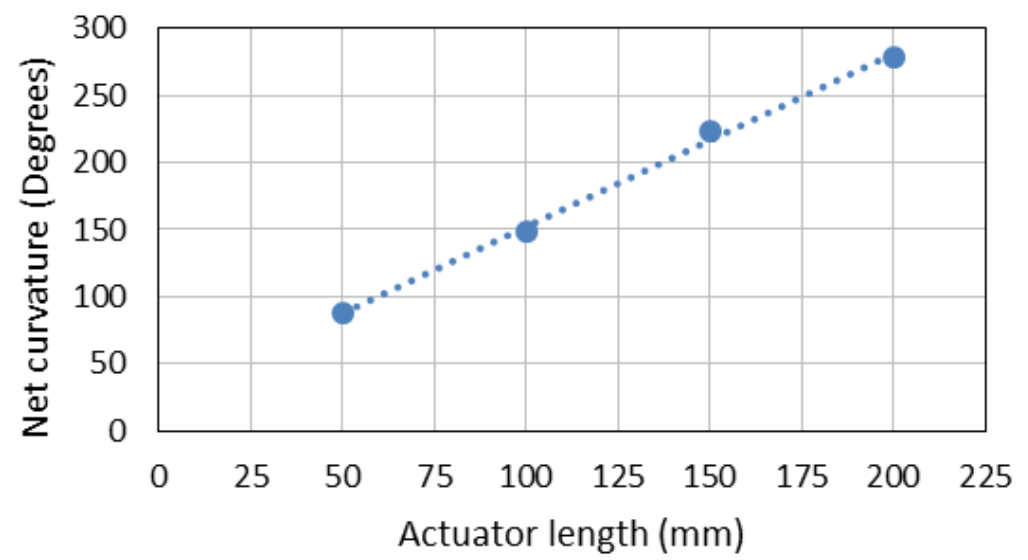

Fig. 7. Maximum curvature versus actuator length at 5 bar gauge pressure

\subsubsection{Pressure - Force}

Due to the soft nature of these actuators, and the constant change of direction in which the force acts, difficulties arise in accurately determining the direct force output. The forces acting against the surface include the circumferential force, from the flexural rigidity of the surface and actuators, resisting bending motion as well as gravitational force acting upon the mass of the surface and the moulding material. An additional consequence of the actuator flexibility is poor performance under shear loading. Initial experimentation sought to equate the actuator to a cantilever beam in which the deflection under a given load would provide a comparative measure of the stiffness over the OPR. The lack of resistance to shear deformation led to bending of the actuators in non-uniform and undesirable curvatures in this setup. Therefore it was decided to use a spring force-gauge to apply a load in a perpendicular direction at the end of the actuator. Figure 9 shows this experimental setup with the actuator clamped and suspended.

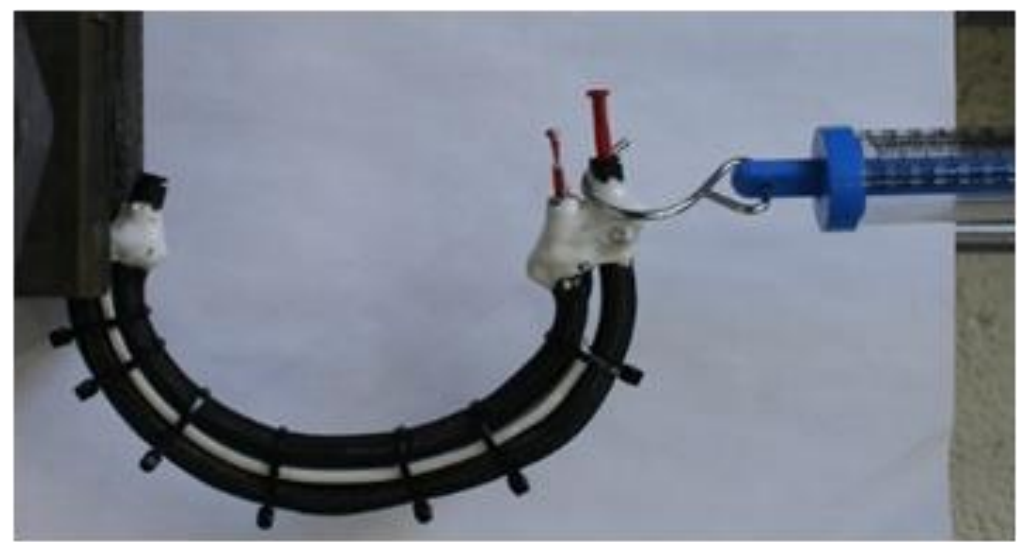

Fig. 8. Experimental setup - loading actuator with spring force-gauge

To characterize the effective force of the actuators against pressure, two experiments with different approaches were devised. The first pressure-force experiment investigated the effect of loading the actuator in a normal direction at the tip of the actuator as shown in Fig. 8. A 1-N constant load was applied via a Newton force-meter and the reduction in curvature from an unloaded actuator was determined at 0.5 bar intervals across the OPR. 
Figure 9 shows the results of this experiment for the $100 \mathrm{~mm}$ and $50-\mathrm{mm}$ actuators. Subtracting the curvatures in these two states gives values for the deflection expected under this load across the OPR. These differences indicate the relative force output of the actuator at a given pressure. They also provide data for the sizing and number of actuators required to support the loads anticipated in intended applications.

The results of the constant-load experiment also show that increasing the length of the actuator increases the expected deflection under a given load at a given pressure. This is to be expected as total curvature is proportional to actuator length according to the results depicted in Fig. 7.

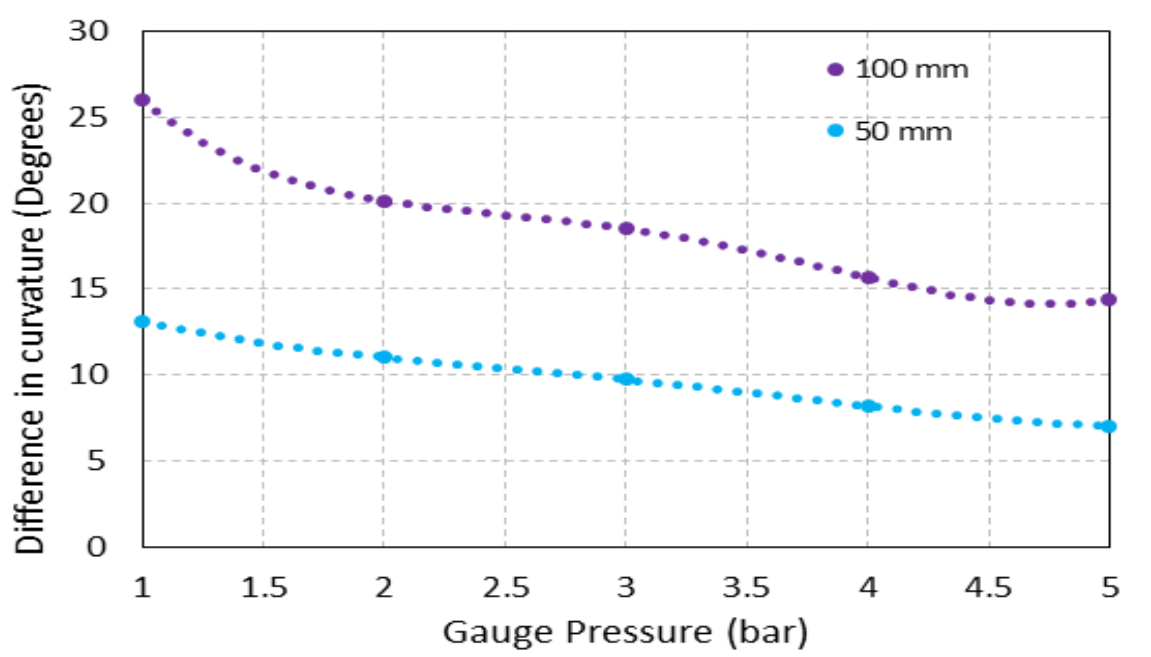

Fig. 9. Difference in actuator curvature between unloaded and 1-N loaded states across operational pressure range for 2 different actuator lengths

In the second experiment, however, the curvature deflection was kept constant by varying the load applied by the Newton force-meter against the pressure increasing up to 5 bars. Results using the $100 \mathrm{~mm}$ actuator for three different curvature deflections are shown in Fig. 10. The results of the second experiment shown in Fig. 10 indicate an increasing resistance and hence rise in force output as the pressure increases.

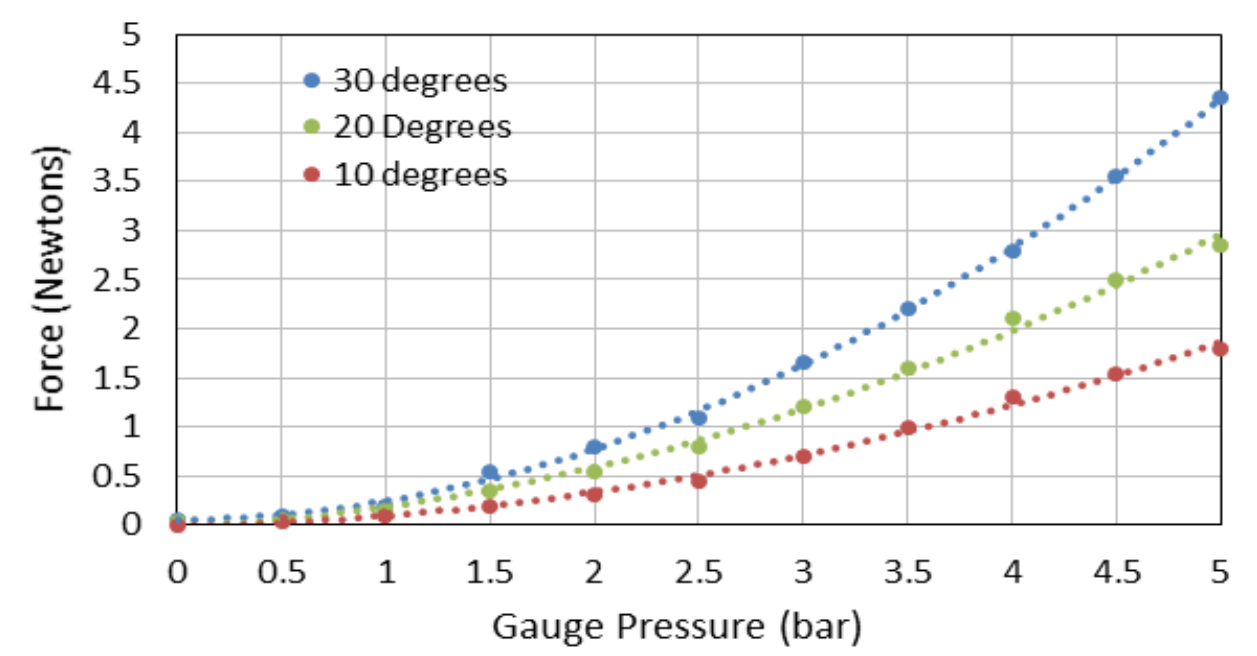

Fig. 10. Force required for 10, 20 and 30 degree differences in deflection between loaded and unloaded states across operational pressure range (100-mm actuator)

For integration into a reconfigurable surface, the results in Fig. 10 along with the pressurecurvature results in Fig. 6 enable a means of determining the pressure required to achieve a 
desired curvature, and the increase in pressure required to maintain that curvature under a given load at various actuator lengths.

\section{Vacuum-jammed surface optimisation}

To achieve the rigidity and stiffness required in the surface for moulding purposes whilst allowing sufficient flexibility to enable reconfiguration, the concept of vacuum jamming was explored. Jamming of granular media within a contained volume allows a transition of the media from a fluid-like state to a solid-like state with increased stiffness. This phase transition has been utilised in several soft robotic applications, notably the work of Cheng et al [16] on the development of a highly articulated manipulator. As such, sufficient literature exists on the mechanics of vacuum jammed granular media. For the reconfigurable mould application explored here, the volume containing the granular medium is a thin, hollow surface with internal ribs, dividing the void into interconnected cells. The optimisation of this element involved assessment of the effect of changes to this internal configuration on the variable stiffness of the surface.

\subsection{Design and fabrication of surfaces}

The surface was designed as a square sheet of silicone of thickness $5 \mathrm{~mm}$. The granular medium was densely packed within an internal void $3 \mathrm{~mm}$ in height. To prevent the medium shifting excessively and maintain a homogeneous fill within the surface, an internal pattern of ribs was required. This segmented the internal void into cells (with cut-outs to allow air flow) and ensured a constant thickness was maintained across the surface during deformation of the surface by the actuators.

To determine the optimal void-rib configuration patterns were designed and cut into $3 \mathrm{~mm}$ thick acrylic sheets with a laser cutter to make moulds (Fig. 11.1). The shell of the surface was then cast from these using condensation cure RTV silicone (Fig. 11.2). After de-moulding and de-flashing the shells (Fig. 11.3), the filtered vacuum fitting was sealed into the surface with silicone (Fig. 11.4). When cured, a granular medium was densely packed inside up to the level of the ribs and external walls (Fig. 11.5). From this a flat $1 \mathrm{~mm}$ surface was cast, and when semi-cured placed onto the top of the shell. The surface was then fully cured under pressure to ensure consolidation between the internal ribs and the top surface (Fig. 11.6). Initial experimentation with this manufacturing method involved alternate approaches to sealing the top of the shell such as painting silicone onto the contact points and using a fully cured top surface. However, following from the study by Jiang et al [9] on the effect of membrane coupling in vacuum jammed elements, it was concluded that the performance of the surface could be improved through the cohesion of the top layer of the granular medium with the membrane wall.

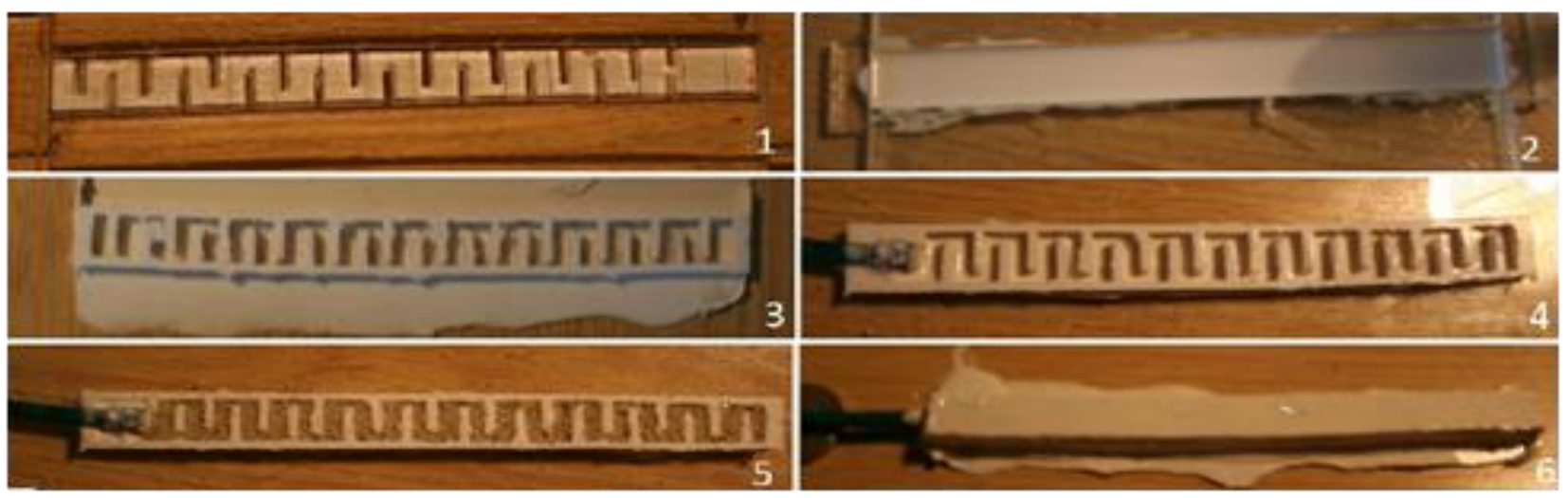

Fig. 11. Vacuum surface manufacture process 


\subsection{Experimental procedure and results}

To evaluate optimal stiffness and deformation criteria, the performance of the surface was considered as the increase in stiffness gained under the maximum negative pressure of a vacuum pump, e.g. when 'jammed'. Parameters that have a significant effect upon the surface performance are as follows: internal configuration and granular medium. The surface thickness, vacuum fitting design, and surface material were kept constant.

Increasing the thickness of the surface will naturally decrease the maximum curvature that can be obtained by the actuator sets. It is also clear that increasing the thickness (with constant wall thicknesses) will increase the volume ratio of granular medium to surrounding volume material and increase the variable stiffness. This is logical as the silicone walls exhibit no increase in stiffness when the surface is jammed and indeed contributes to the elastic resistance during deformation. With consideration to this trade-off between flexibility and maximum stiffness, the surface was kept at a thickness of $5 \mathrm{~mm}$ for all experimentation. The vacuum fitting design geometry is located at a single corner of the surface and therefore will not affect surface stiffness and/or deformation during operation. Material composition of the surface could have a significant effect on stiffness. However, analysing the multitude of possible alternatives was outside of the scope of this paper and therefore silicon was used due to is flexibility and ability to hold under vacuum and external loading conditions.

For the purposes of comparison of the relative stiffness achieved by each surface test when vacuum jammed was undertaken for cantilever beams 100x15 mm. The experimental setup can be seen in Fig. 12, where the surfaces were laid flat on the workbench and vacuum jammed by a pump with strength $1.5 \times 10^{-2}$ Torr. They were then cantilevered from the edge of the workbench and rotational deflection from the horizontal under their own weight in a jammed condition recorded. The surfaces were then unjammed and the deflection recorded again. The difference between these values provided a relative measure of the increase in stiffness obtained, allowing comparison of the effects of the internal configuration and granular medium.

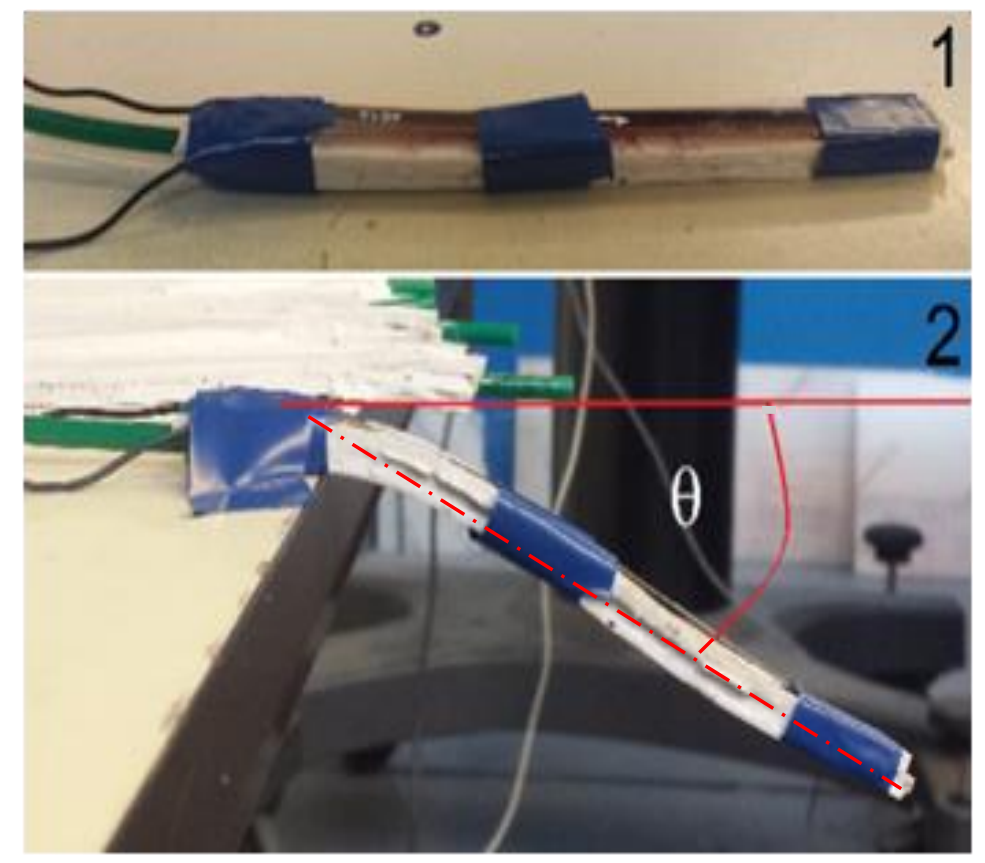

Fig. 12. 1) Attachment of flex sensor to test surfaces, 2) Experimental setup to determine relative stiffness. Cantilevered test surface (unjammed surface) 


\subsubsection{Internal configuration}

Initial experiments investigated the effect of varying the internal geometric configuration on the jamming performance. For these tests, a set of six small 'surfaces', 100mm in length and $15 \mathrm{~mm}$ in width, were fabricated. The important considerations when designing the initial test configurations were to isolate the granular medium into cells (preventing shifting) and to include no uninterrupted lines (maintaining aclear path for the air flow). Another consideration was the proportion of empty volume to the total volume of the shell. As the performance improves with increased filler to membrane ratios, the number of ribs should not be increased beyond that needed to sufficiently constrain the granular medium. The surface profile and stiffness isotropy are related to the choice of geometric shape used for the ribs. To achieve greater uniformity in both the profile and flexibility of the surface, elements of symmetry were included in the designs, whilst avoiding uninterrupted channels. Figure 13 shows the geometric configurations explored, with an empty shell (no internal ribs shown in surface 6) being used as a control.

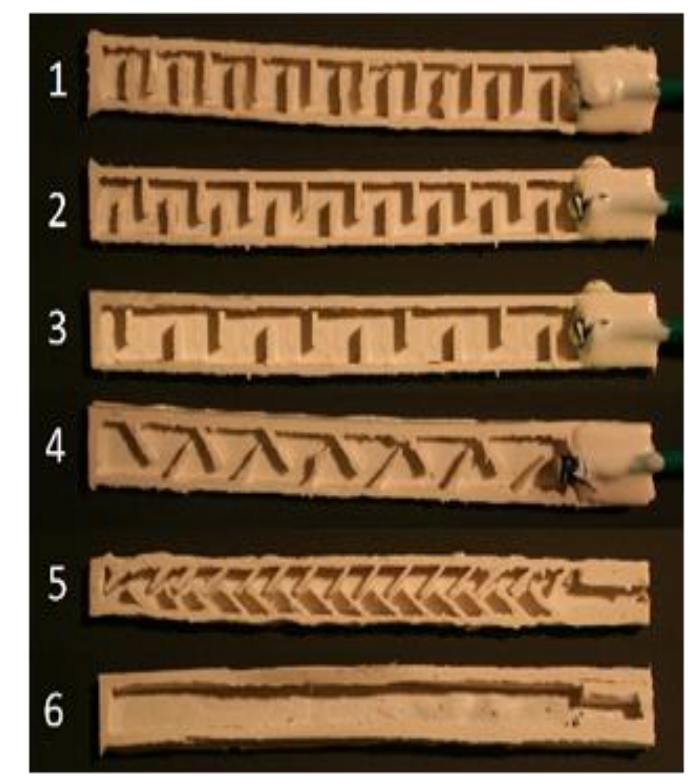

Fig. 13. Initial test surfaces with varying internal configurations

The set explored three geometries: alternating direction $45^{\circ}$ ribs (surface 4; Fig. 13), unidirectional $45^{\circ}$ ribs (surface 5; Fig. 13) and orthogonal ribs (surfaces 1, 2 and 3; Fig. 13). For all surfaces, the rib thickness was kept constant at $1.5 \mathrm{~mm}$. In surfaces 1,2 and 3 , the rib spacing (cell size) was varied from 3 to 5 to $7 \mathrm{~mm}$ respectively.

Table 1 shows the results from the initial vacuum bag configuration experiment. From this, surface 2 exhibited the largest difference in deflection between the jammed and unjammed states $\left(12^{\circ}\right)$. The results suggest that orthogonal configurations (square sided cells) are the best choice for the internal geometry with surfaces 1 and 2 outperforming the other configurations in terms of largest difference between jammed and unjammed conditions. The effect of the rib spacing on performance can be seen through comparison of surfaces 1-3. The optimum spacing in this case is somewhere around $5 \mathrm{~mm}$. As this test set only covers a small range, the results are not conclusive. However, for the purposes of a small scale reconfigurable mould cell sizes of $5 \mathrm{~mm}$ provided suitable continuity in the surface as it forms curves, while maintaining an acceptable surface profile. 
Table 1: Comparison of jammed and unjammed deflections for differing internal configurations (the numbers 1-6 correspond to the test results using configurations numbered in Fig. 13)

\begin{tabular}{|c|l|c|c|c|}
\hline $\begin{array}{c}\text { Test } \\
\text { No. }\end{array}$ & \multicolumn{1}{|c|}{ Internal geometry } & $\begin{array}{c}\text { Unjammed } \\
\text { deflection } \\
\text { (degree) }\end{array}$ & $\begin{array}{c}\text { Jammed } \\
\text { deflection } \\
\text { (degree) }\end{array}$ & $\begin{array}{c}\text { Difference } \\
\text { (degree) }\end{array}$ \\
\hline 1 & Orthogonal ribs, 1.5mm thickness, 3mm spacing & 66 & 57 & 9 \\
\hline 2 & Orthogonal ribs, 1.5mm thickness, 5mm spacing & 66 & 54 & 12 \\
\hline 3 & Orthogonal ribs, 1.5mm thickness, 7mm spacing & 66 & 62 & 4 \\
\hline 4 & Alternating direction 45 ribs, 1.5mm thickness, 7.5mm spacing & 61 & 55 & 6 \\
\hline 5 & Unidirectional 45 ${ }^{0}$ ribs, 1.5mm thickness, 5mm spacing & 70 & 68 & 2 \\
\hline 6 & Empty void, no internal ribs & 75 & 69 & 6 \\
\hline
\end{tabular}

\subsubsection{Granular medium}

A key variable in the performance of the vacuum jammed surface is the choice of granular medium. A good deal of literature exists on the mechanics of structurally jammed granular media. At comparative levels of compressive stress, the stiffness is governed by the relative motion ability of the fluid media. This is dependent on particle shape, size, density, material, homogeneity and surface texture [10]. From results presented by Jiang et al [9] and Cheng et al [16] along with additional comparative data on the performance of various granular media, three media that were known to perform well were selected for further comparison. The jamming strength of finely ground coffee, fine sand and very fine sand were compared using the control surface with no internal configuration (number 6 in Fig. 13).

Using the best performing surface (number 2 in Fig. 13), tests to determine the best medium were accomplished. Tests 1, 2 and 3 in Table 2 correspond to the ground coffee, fine sand and very fine sand mediums respectively.

Table 2: Comparison of jammed and unjammed deflection for three granular media (finely ground coffee for tests 3 and 6 , fine grade sand for tests 1 and 4, and very fine grade sand for tests 2 and 5)

\begin{tabular}{|c|c|l|c|c|c|}
\hline Test No. & $\begin{array}{c}\text { Surface configuration } \\
\text { (Number in Fig. 13) }\end{array}$ & $\begin{array}{c}\text { Granular } \\
\text { Medium }\end{array}$ & $\begin{array}{c}\text { Unjammed deflection } \\
\text { (degree) }\end{array}$ & $\begin{array}{c}\text { Jammed deflection } \\
\text { (degree) }\end{array}$ & $\begin{array}{c}\text { Difference } \\
\text { (degree) }\end{array}$ \\
\hline 1 & 2 & Fine Sand & 76 & 72 & 4 \\
\hline 2 & 2 & Super-fine sand & 66 & 57 & 9 \\
\hline 3 & 2 & Fine Coffee & 66 & 54 & 12 \\
\hline 4 & 6 & Fine Sand & 69 & 65 & 4 \\
\hline 5 & 6 & Super-fine sand & 68 & 63 & 5 \\
\hline 6 & 6 & Fine Coffee & 65 & 59 & 6 \\
\hline
\end{tabular}

For control comparison purposes the surface with no internal ribs (i.e. number 6 in Fig. 13) is shown for ground coffee, fine sand and very fine sand mediums as tests 4-6 in Table 2 respectively. The relative stiffness and strength to weight ratio of these three granular media were then compared to find the optimum choice for this application by measuring the amount of deflection under jammed versus unjammed conditions. Results in Table 2 show that finely ground coffee (tests 3 and 6) outperforms both fine grade sand (tests 1 and 4) and very fine grade sand (tests 2 and 5). This is, in part, due to the significantly higher density, and therefore greater mass, of the sands affecting displacement under gravity in comparison to the coffee. From these results, finely ground coffee was chosen as the granular medium for use in subsequent surfaces. 


\section{Prototype reconfigurable moulding tool}

Having experimentally characterised the project's main components i.e. the PAM and jamming surface, the two elements were consolidated into prototype reconfigurable surfaces. This involved the design of two enlarged vacuum surfaces with difference in surface's size, and number and arrangement of actuators leading to distinct functions. In the following, the characteristics of each reconfigurable surface will be explained. The first case will be also followed by presenting the results of a numerical analysis carried out for this arrangement to picture and verify the performance of the experimentally developed prototype.

\subsection{Reconfigurable system 1: (surface integrated with 2 pairs of PAM sets)}

\subsubsection{Design, fabrication and performance of reconfigurable system 1}

The first system of dimensions $160 \times 180 \mathrm{~mm}$ comprised of a $160 \mathrm{~mm}$ square surface (with internal cells) and two 10mm width tabs of pure silicone along the length of opposite edges for attachment of the actuators. This 'double-sided parallel muscle' arrangement allows the formation of single axis, symmetrical 3-D shapes of uniform curvature as well as shapes with inverted curvatures at either axis. The parameters for the internal configuration were chosen from the experimental results. As surface 2 in Fig. 13 performed the best, orthogonal ribs with thickness $1.5 \mathrm{~mm}$ and spacing of $5 \mathrm{~mm}$ were used. Scaling up the width of the surface from the initial design necessitated the inclusion of additional cut-outs in the ribs to allow unimpeded air flow throughout the surface. The spacing and width of these cut-outs were assumed to have negligible effect on the surface performance, provided that the vacuum was able to carry throughout. They were therefore set at $1.5 \mathrm{~mm}$ and $5 \mathrm{~mm}$ as these spacing proved suitable in the initial experiment set. Also, finely ground coffee was used as the granular medium based on the conclusion inferred from the data in Table 2.

The fabrication process is shown in Figs. 14. The sub-processes for the vacuum surface (Fig.14a) and actuators followed the steps outlined in sections 3.1 and 4.1. To form the actuator sets, PAMs were joined to the surface along the silicone tabs at each edge. The $10 \mathrm{~mm}$ width PAMs were sewn above and below the tab at 20mm intervals (Fig. 14b). They were securely joined to each other at the fittings to prevent detachment and force deformation around a curvature. For the purposes of feedback and control, flex sensors were sealed into the surface with silicone. The positioning was equidistant about the central axis, at the inside edge of each actuator. A thin silicone sheet was then cast and moulded around each actuator set to maintain uniformity of curvature as the actuators are pressurised (Fig. 14b). The sheet also provides a cover for the PAMs and sensor and defines the borders of the working section of the mould surface. 

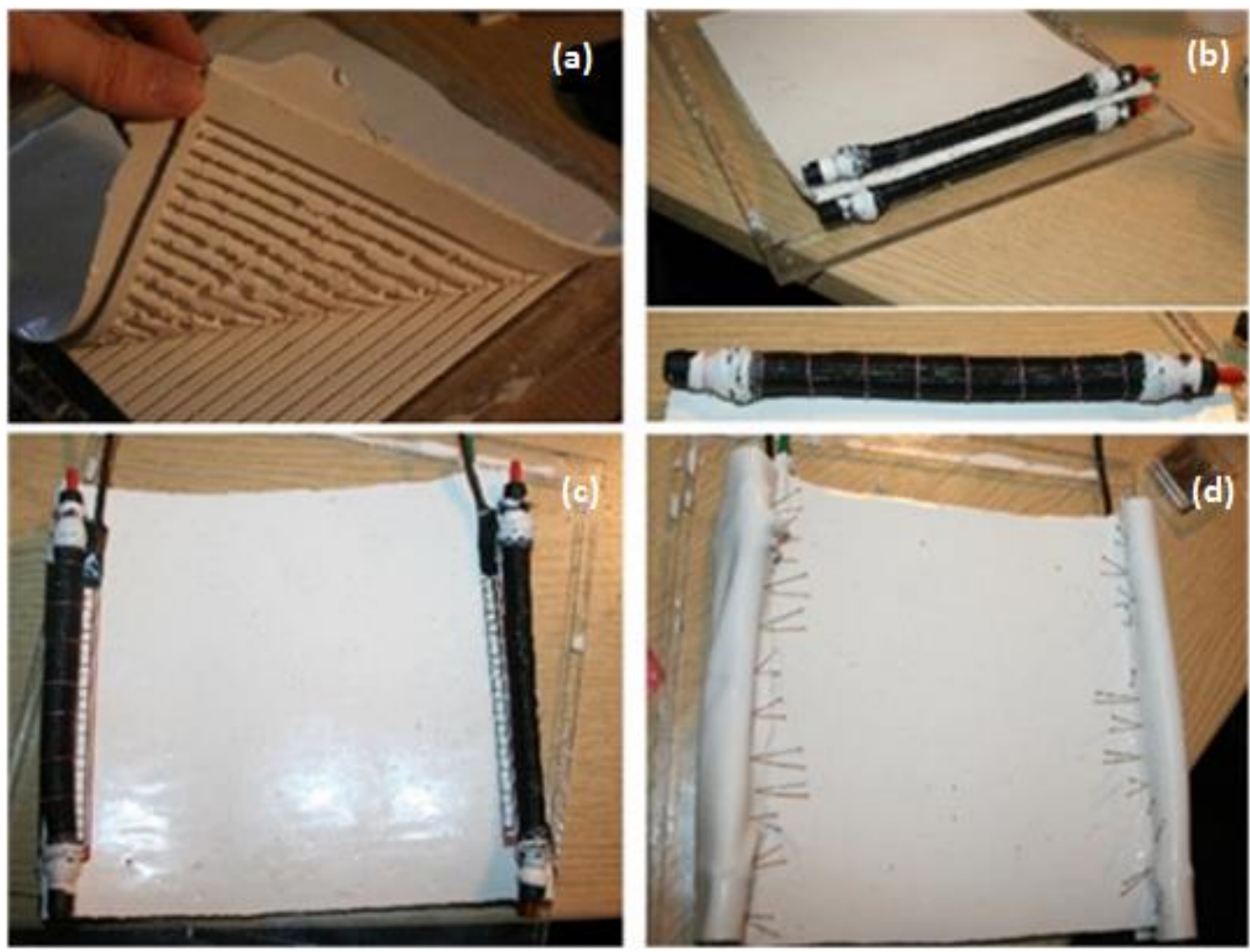

Fig. 14. Fabrication process for reconfigurable moulding system 1 with 2 pairs of PAM sets

As can be seen from different views in Figs. 15, the integrated system performed well in operation, forming uniform and equal curvature at both actuators as expected. The pressurecurvature graph recorded for the surface through this experiment is depicted in Fig. 16.
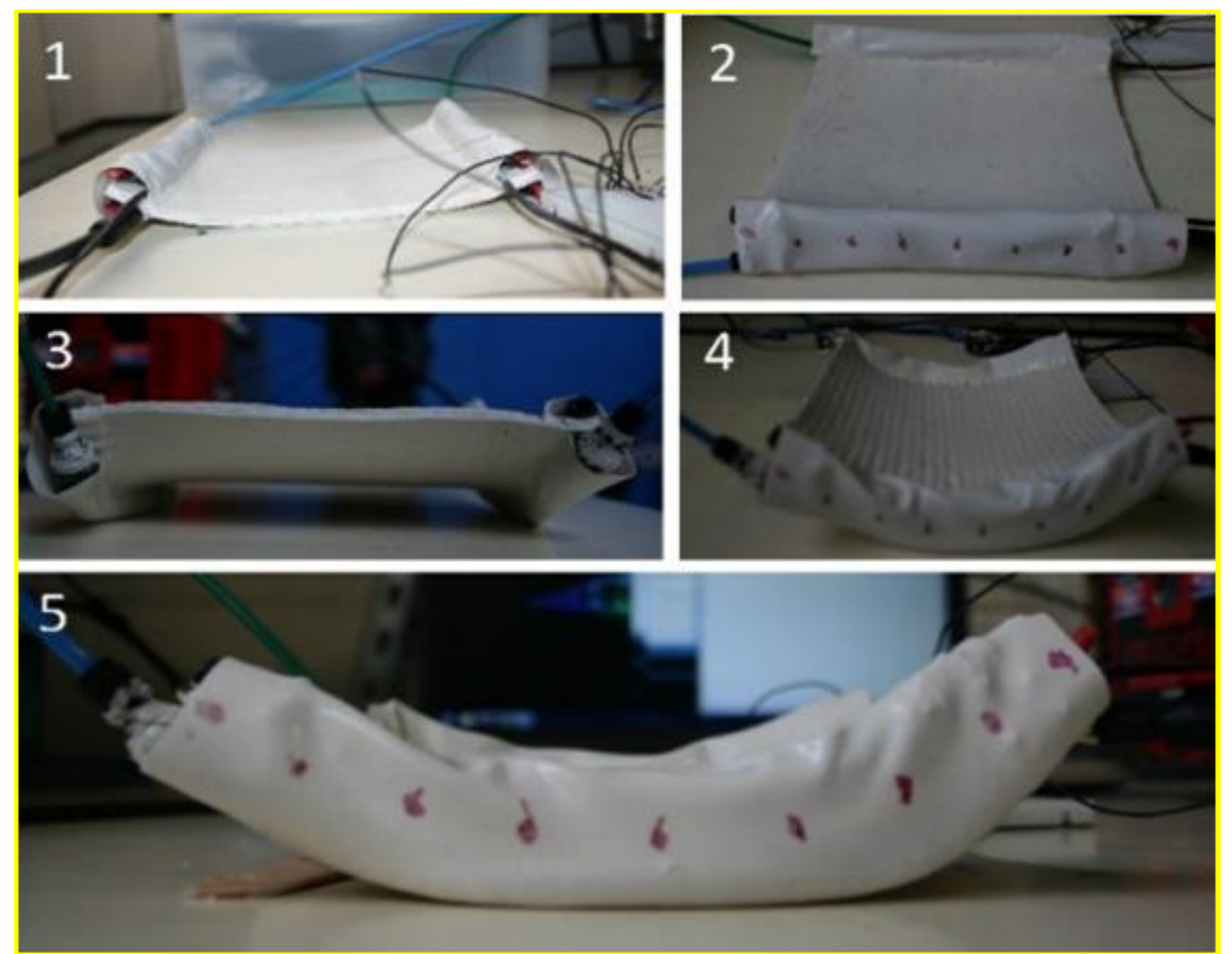

Fig. 15. Reconfigurable system, unactuated ( 1 and 2$)$ and at $80^{\circ}$ curvature $(3,4$ and 5$)$ 


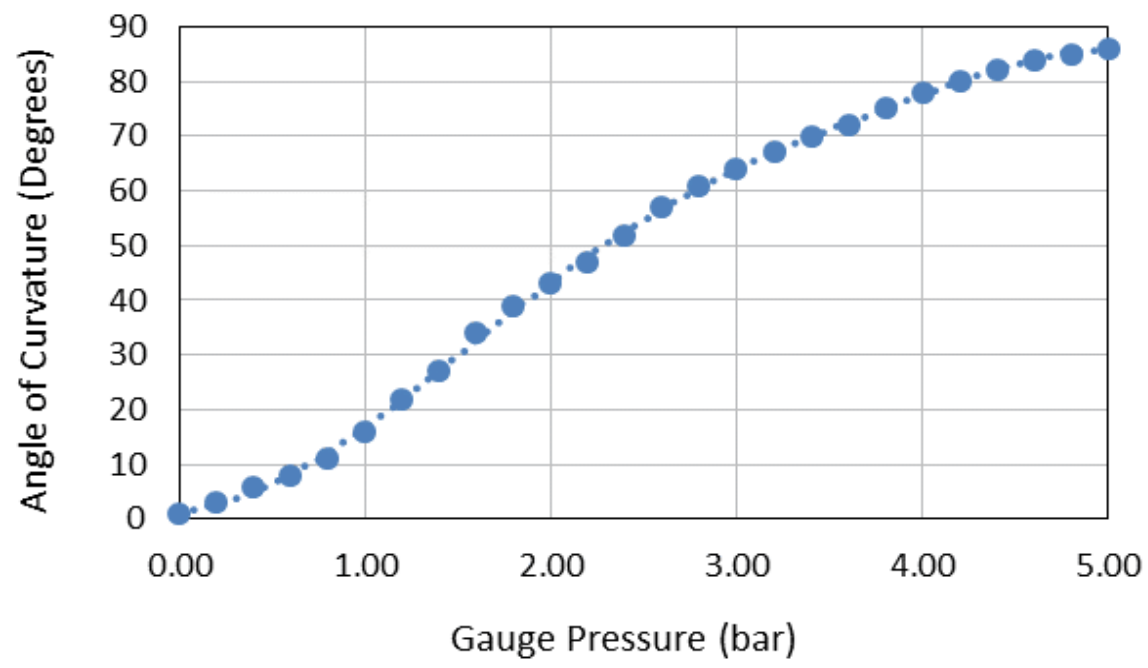

Fig. 16. Pressure-curvature characteristics of reconfigurable system 1

\subsubsection{Developing a numerical model for reconfigurable system 1}

To evaluate the performance of the surface in terms of its ability to recreate a shape of uniform curvature, a MatLab code was developed to generate a 3-D surface plot numerically based on the experimental pressure-curvature data. The model takes inputs of the surface dimensions and pressures at each actuator and converts the pressures to curvatures. Modelling the actuators as arc segments of a larger circle, the actuator lengths (arc circumferences) are used to calculate the effective radii of these circles. From this, the chord lengths are calculated. These represent the axial lengths of the surface (distance between end points in x-direction), which reduce as the surface curves. Arcs of the specified curvature are then plotted along the lines of each actuator. A MatLab function is used to carry out a linear interpolation (using Delaunay triangulation) between the two arcs across the width of the surface. This allows a smooth plot of the surface to be modelled at both equal and non-equal curvatures as shown in Fig. 17.

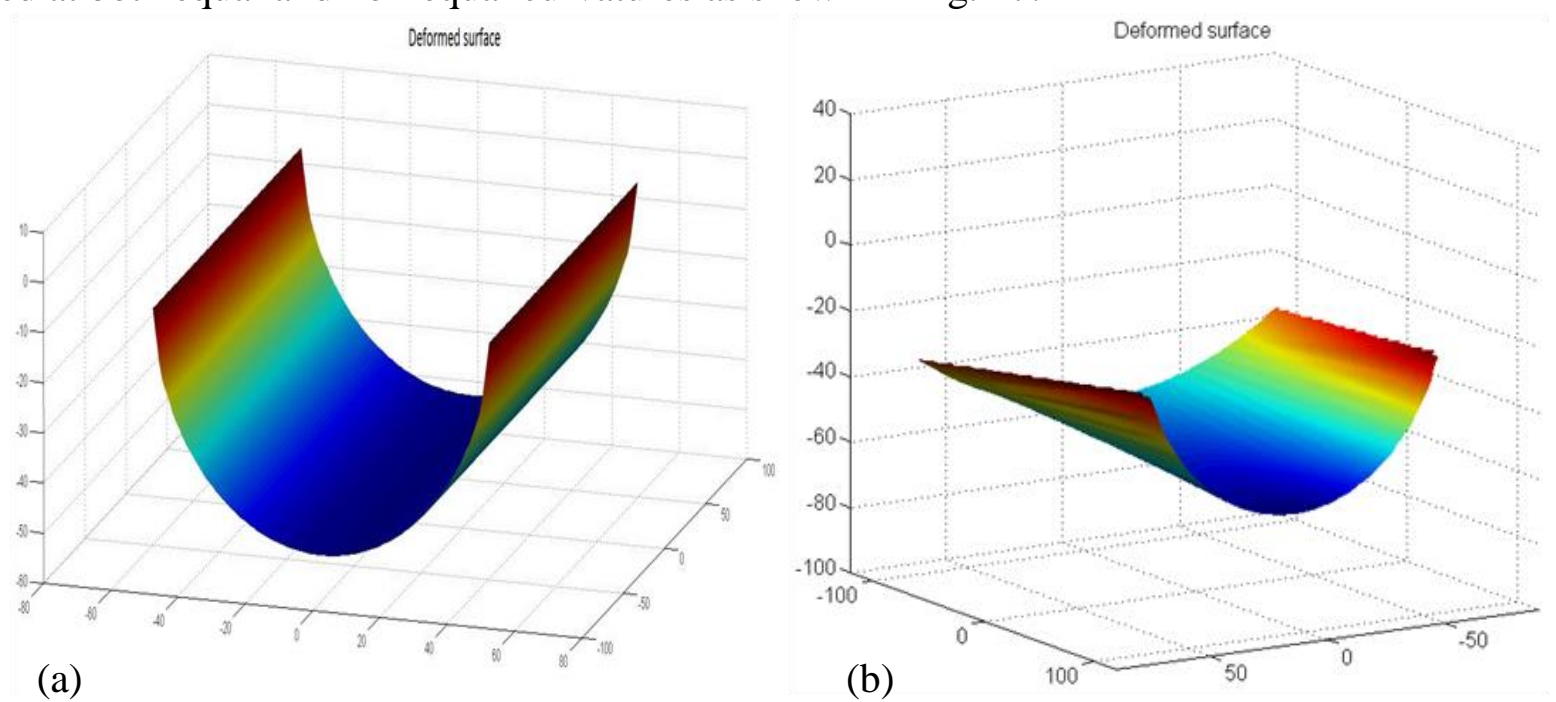

Fig. 17. Numerically generated surface models for system 1: a) $180^{\circ}$, b) $60^{\circ}$ and $140^{\circ}$

To compare the experimental surface against its numerical model, 2-D projection of the curvature at the actuators was carried out using side profile photos (shown in Fig. 15.5) and a modified surface model script. Figures 18 (a) and (b) depict comparisons of the 2-D surface model and the physical surface at two angles of curvature. It can be seen that the shapes formed by the 
actuators match closely to the model, forming almost perfectly uniform arcs of curvature. Therefore, in conjunction with Figs. 15.3-15.5, the surface can be assumed to perform adequately in its assumption of single axis, symmetrically curved shapes. The trivial error in the model can be partly accounted for by the lack of consideration of the force interactions.

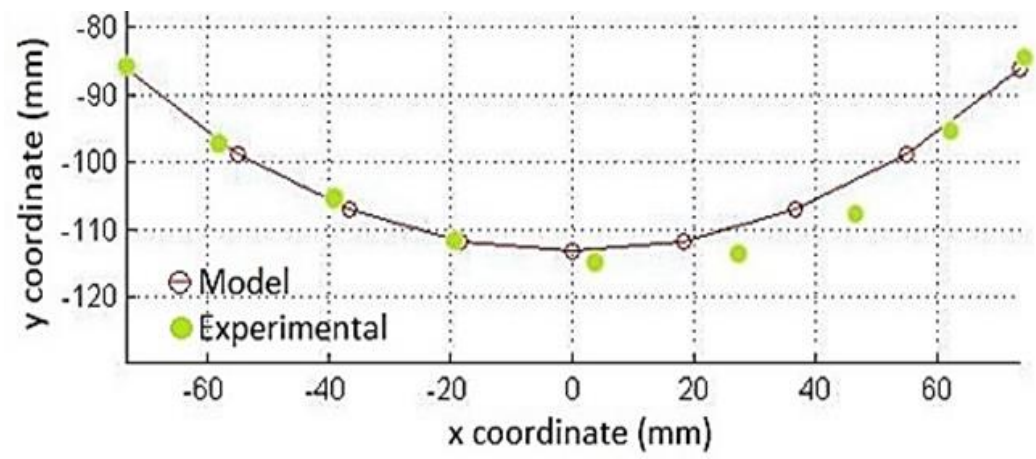

(a)

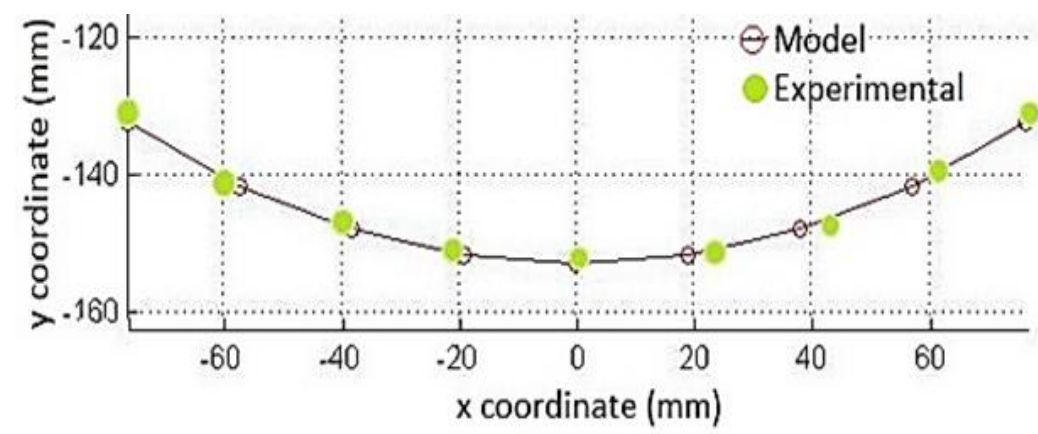

Fig. 18. Comparison of model and experimental curvature for system 1 at: a) $80^{\circ}$, b) $60^{\circ}$

\subsection{Reconfigurable system 2: (surface integrated with 8 pairs of PAM sets)}

Having reconfigurable system 1 designed, operated and verified numerically, it was desired to determine a configuration of the muscles that would lead to the most flexibility of achievable shapes. For this reason, the second reconfigurable system was designed and fabricated which composed of two bi-directional muscles per edge of the square surface (16 independent muscles in total). This application is aimed to underlie the development of a controllable, reconfigurable surface system that is able to configure multiple-curvature surfaces for developing industrial-scale forming of composite sheets.

\subsubsection{Characteristics of reconfigurable system 2}

The second moulding system included a larger surface than the first system with total dimensions $280 \times 280 \mathrm{~mm}$. The properties for the surface internal configuration such as orthogonal ribs thickness, spacing gaps, cut-outs and granular medium were chosen the same as those applied for the first system. Figure 19 illustrates the arrangement for prototype moulding system 2 . The pen in this figure is shown as a scale to visualise the system actual size. 


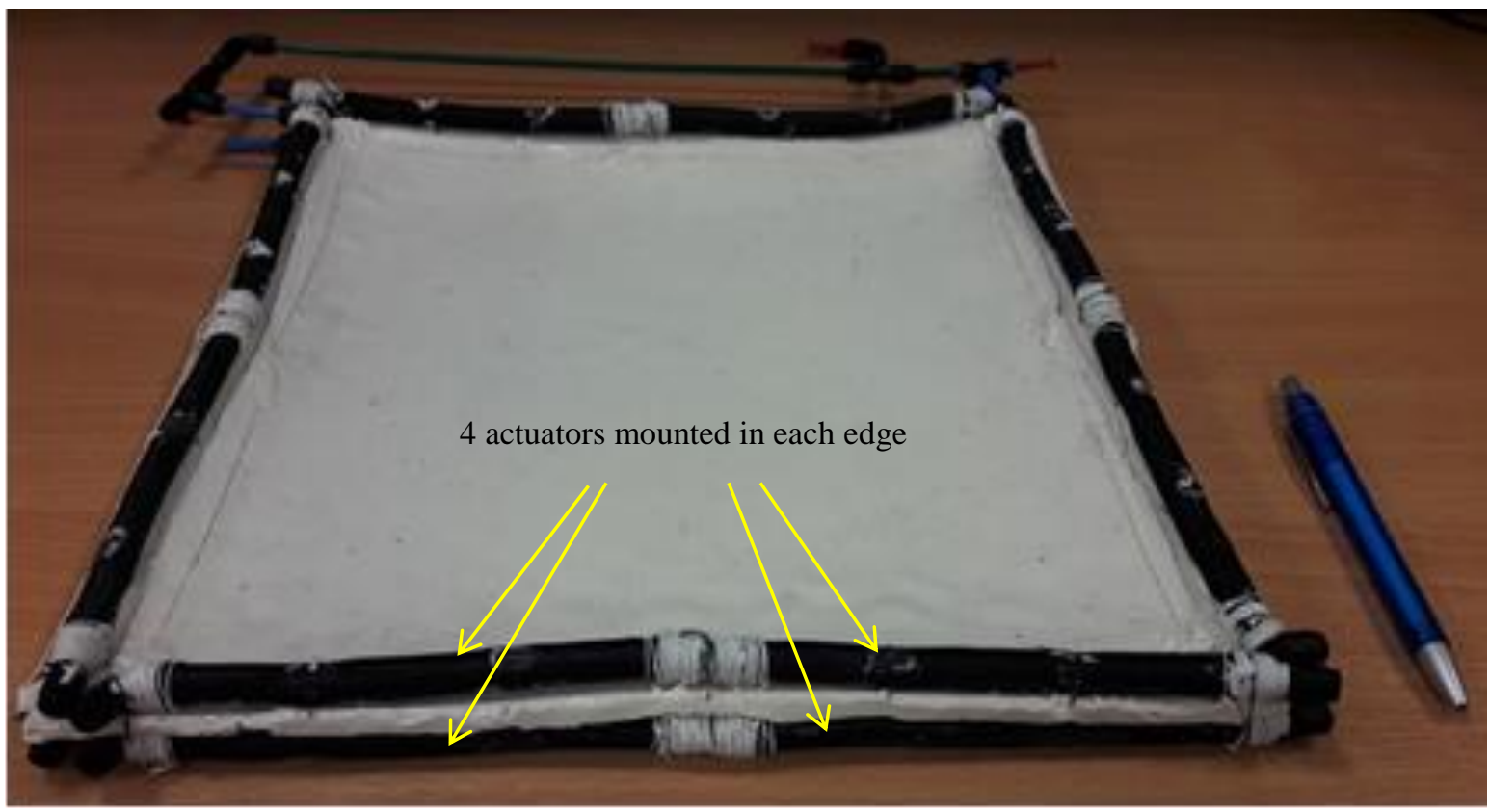

Fig. 19. Prototype moulding system 2 supplied by two pairs of PAM sets in each side of the square surface.

Fabrication of the second configuration was completed by casting and moulding a thin covering silicone sheet around the actuator arrangement to keep the evenness of curvature when actuators bend. This process along with the final integrated system are shown in Fig. 20a and Fig. 20b.

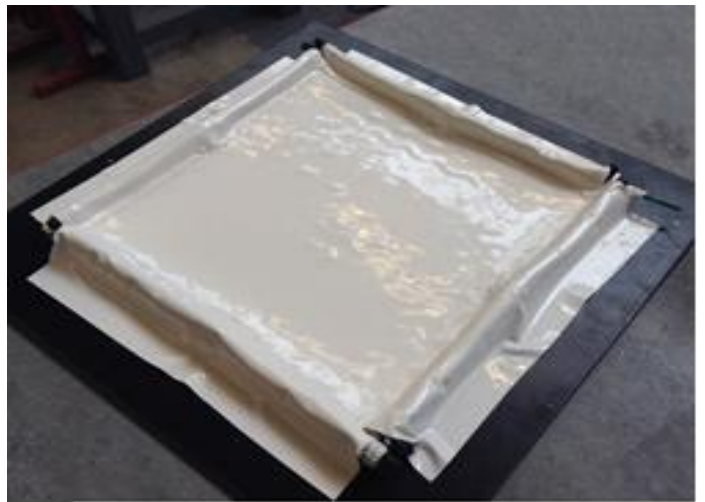

(a)

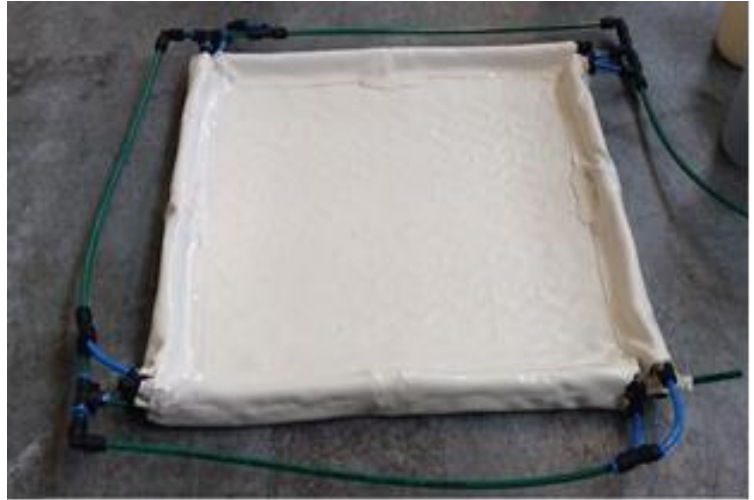

(b)

Fig. 20. Reconfigurable moulding system 2; a) surface in process to be covered and moulded by a silicon sheet, b) final setup.

\subsubsection{Reconfigurable system performance}

The fabricated surface shown in Fig. 20.b supplied by 8 pairs of PMA sets was then able to be deformed into different complex profiles through operating actuators on different patterns at certain pressures. Once in the desired shape the trapped air in the surface was sucked out straightaway by a vacuum pump, thus stiffening the surface representing. An example of the stiffened surface when the actuators are removed is shown in Fig. 21. Further stiffness is then present at interface points with the actuators if they are still pressurized. This further strengthens the edges of the surface, and in future actuation pairs could be integrated across the surface to increase potential deformation complexity, as well as provide additional stiffness under loading. 


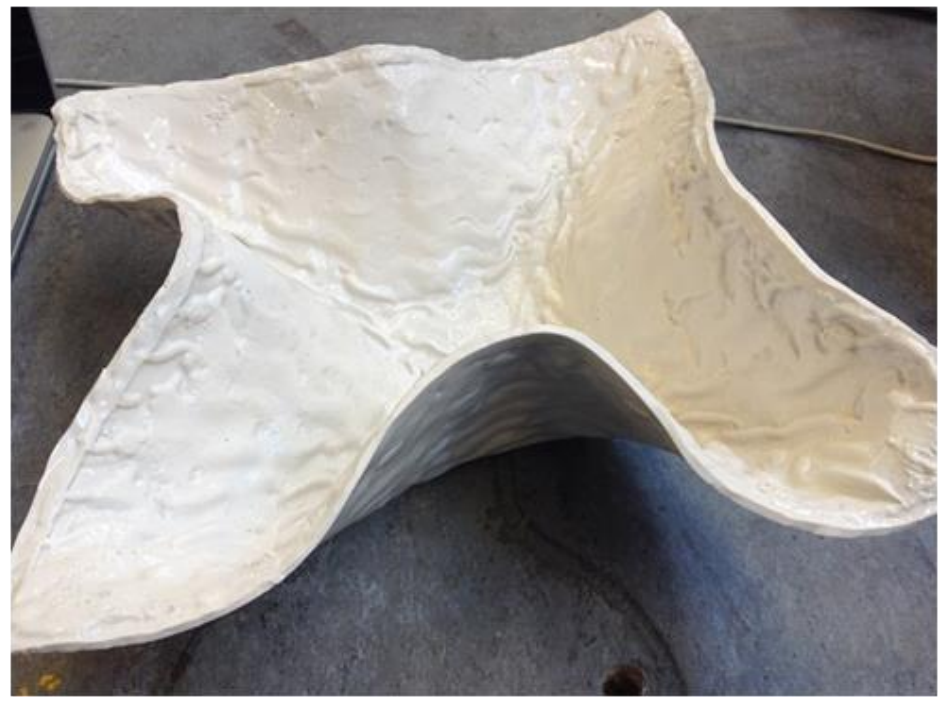

Fig. 21: A sample test of a vacuum-jammed surface

To demonstrate a moulding process, absorbent paper previously dipped in a wax melting pot were placed on the stiffened surface and allowed to set. The idea is that the absorbent papers absorb wax while the wax solidifies shortly after coming out of the melting pot. Therefore, a sufficient number of the dipped papers in the wax covering the stiffened surface roughly represent a sheet formed on the surface using the "wet layup" process in composite material construction in terms of resulting temperature and weight. Alternative deposition processes are also possible, and future work will look at redesigning the surface and actuators to accommodate use within an autoclave environment to further enhance use. Figure 22 shows the process as wax papers are being applied to a surface (a), and the resulting surface without the mould support afterwards (b). Further surface profiles created by the reconfigurable mould due to its potential for achieving different actuation patterns of the muscles are shown in Fig. 22 (c-e). The results show that such a system would have many advantages as other reconfigurable moulding systems such as flexibility, quickness and adaptability, while additionally benefitted from being very light, low cost, versatile and taking up less space than pin-based systems. 


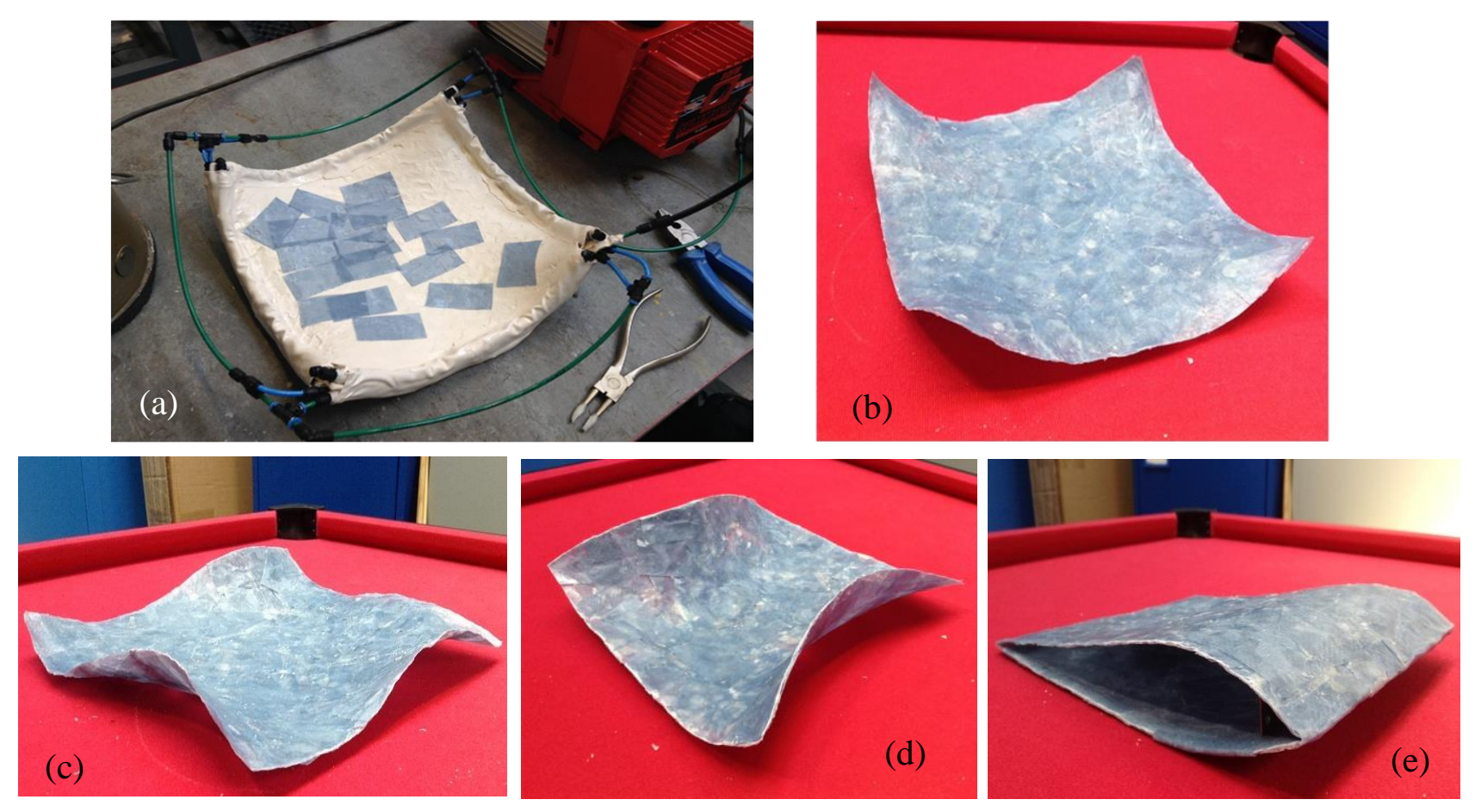

Fig. 22. a) Prototype moulding system 2 under process as wax papers are being applied to the jammed surface; b) the moulded wax surface representing a sample profiled sheet of multiple-curvature; c-e) three further surface profiles formed by the reconfigurable mould.

\section{Concluding remarks}

This paper has presented the design optimisation of a pneumatically actuated, vacuum jammed continuum surface and explored the potential for its use as a reconfigurable mould. Characterisation of the actuation element's force-length-curvature profiles and surface stiffness under jamming evaluated and used to demonstrate the ability to form uniform curvatures suitable for moulding. This system compared to other reconfigurable moulding systems is very light, costeffective, and rapidly operated with little occupying space.

Two prototype samples for this reconfigurable moulding tool were produced - using respectively 4 and 16 PAMs arranged in pairs - and tested to show the adaptability of the system and demonstrate practical implementation for creating surfaces with either simple or complex profiles of smooth curvatures. The first sample (the one with 4 actuators) was also provided by a numerical model to picture the surface and evaluate the experimental results. This work underlies future effort to develop an industrial scale prototype of the system with integrated sensors to produce and maintain surface mould shapes for use in the forming of fibre carbon composite sheets. Other potential uses for such variable stiffness, reconfigurable systems and their related control models extends to numerous different areas of engineering, from deformable surfaces to soft, compliant end effectors and manipulators for use in automotive, aerospace, manufacturing and healthcare.

\section{Acknowledgement}

This research is funded by the UK Engineering and Physical Sciences Research Council (EPSRC) under grant number EP/N022505/1. 


\section{References}

[1] Weitao, M., Cost modelling for manufacturing of aerospace composites. MSc Thesis, School of Applied Sciences, Cranfield University, 2011.

[2] Peters, B and Marion, E., Design and fabrication of a digitally reconfigurable surface. MSc Thesis, Department of Mechanical Engineering, Massachusetts Institute of Technology, 2011.

[3] Koc, B., and Thangaswamy, S., Design and analysis of a reconfigurable discrete pin tooling system for molding three-dimensional free-form objects, Robotics and Computer-Integrated Manufacturing 27 (2011) 335-348.

[4] Cortés, J., Varela-Jimenez I. and Bueno-Vives M., Reconfigurable tooling by using a reconfigurable material. Chapter in Book: Manufacturing System by Dr. Faieza Abdul Aziz (Ed.), InTech, 2012.

[5] Gibbons, G.J., Segui-Garza J.J., and Hansell R.G., Low-cost resin infusion mould tooling for carbon fibre composites manufacture, IMechE Journal of Aerospace Engineering, Vol. 224(4), 2010, pp 511-518.

[6] Walczyk DF, Lakshmikanthan J, Kirk DR. Development of a reconfigurable tool for forming aircraft body panels, Journal of Manufacturing Systems 1998; 17(4):287-96.

[7] Walczyk DF, Im YT. A hydraulically-actuated reconfigurable tool for flexible fabrication: implementation and control, Journal of Manufacturing Science and Engineering 2000; 122(3):562-8.

[8] Im YT, Walczyk DF, Schwarz RC, Papazian JM. A comparison of pin actuation schemes for large-scale discrete dies, Journal of Manufacturing Processes 2000; 2(4):247-57.

[9] Jiang, A., Design of a variable stiffness flexible manipulator with composite granular jamming and membrane coupling. IEEE International Conference on Intelligent Robots and Systems. Algarve, Portugal, October 2012.

[10] Khalifa, S., Mechanics of jammed media used as a structural element. MSc Dissertation, Massachusetts Institute of Technology, 2012.

[11] Steltz, E., Mozeika, A., Rembisz, J., Corson, N., and Jaeger H.M., Jamming as an enabling technology for soft robotics. In Proceedings of SPIE - The International Society for Optical Engineering 7642, Electroactive Polymer Actuators and Devices (EAPAD), San Diego, California, USA, March 2010. doi:10.1117/12.853182.

[12] Walczyk, D., Rapid and reconfigurable tooling methods for manufacturing large-scale plastic and composite parts. Presentation, Center for Automation Technologies and Systems, 2010. [Online] Available at: http://www.case2010.org/TVES07Presentations/TVES\%2007\%20Walczyk.pdf

[13] Chou, C.P., and Hannaford B., Measurement and Modelling of Mckibben Pneumatic Artificial Muscles, IEEE Transactions on Robotics and Automation,Vol. 12, No.1, February 1996.

[14] Daerden, F. and Lefeber, D., Pneumatic Artificial Muscles: Actuators for Robotics and Automation. European Journal of Mechanical and Environmental Engineering 47(1), March 2002.

[15] Shepherd RF1, Ilievski F, Choi W, Morin SA, Stokes AA, Mazzeo AD, Chen X, Wang M, Whitesides GM., Multigait soft robot, Proc Natl Acad Sci U S A, 108(51):20400-3, 2011 Dec 20; doi: 10.1073/pnas.1116564108. 
[16] Cheng, N.G., Lobovsky, M.B., Keating, S.J., Setapen, A.M., Gero, K.I., Hosoi, A.E., and Iagnemma, K.D., "Design and analysis of a robust, low-cost, highly articulated manipulator enabled by jamming of granular media", IEEE International Conference on Robotics and Automation. Minnesota, USA, pp. 4328-4333, May 2012.

[17] Majidi, C., Shepherd. R.F., Kramer R.K., Whitesides G.M., and Wood R.J., "Influence of Surface Traction on Soft Robot Undulation", The International Journal of Robotics Research 32(13) 1577-1584, 2013.

[18] Stanley, A.A. and Okamura, A.M., Controllable Surface Haptics via Particle Jamming and Pneumatics, IEEE Transactions on Haptics, vol. 8, no. 1, January-March 2015.

[19] Delson, N., Hanak T., Loewke K., and Miller D.N., Modeling and implementation of Mckibben actuators for a hopping robot. In: Proceedings in IEEE International Conference in Advanced Robotics, 2005.

[20] Kang, R, Guo, Y, Chen, L, Branson III, DT, and Dai JS, Design of a pneumatic muscle based continuum robot with embedded tendons. IEEE/ASME Transactions on Mechatronics 22.2 (2017): 751-761.

[21] McMahan, W, Chitrakaran, V, Csencsits, M, Dawson, D, Walker, ID, Jones BA, Pritts, M, Dienno, D, Grissom, M, and Rahn CD, Field trials and testing of the OctArm continuum manipulator. Robotics and Automation, in Proceedings 2006 IEEE International Conference on. IEEE, 2006. 